\title{
Complications of cataract surgery in Wistar rats undergoing treatment with tamsulosin
}

\author{
RALUCA MARIA POPESCU ${ }^{1}$, CIPRIAN OBER ${ }^{2}$, BOGDAN SEVASTRE $^{2}$, MARIAN TAULESCU $^{3}$, \\ MIHAI NEGRU $^{3}$, IULIA MELEGA ${ }^{3}$, SIDONIA BOGDAN ${ }^{2}$, CRISTINA NICULA ${ }^{1}$ and IOAN COMAN ${ }^{1}$ \\ ${ }^{1}$ Department of Surgical Specialties, Faculty of General Medicine, 'Iuliu Haţieganu' University of Medicine \\ and Pharmacy, 400012 Cluj-Napoca; ${ }^{2}$ Clinical and ${ }^{3}$ Paraclinical Departments, Faculty of Veterinary Medicine, \\ University of Agricultural Sciences and Veterinary Medicine, 400372 Cluj-Napoca, Romania
}

Received April 4, 2018; Accepted October 2, 2018

DOI: $10.3892 /$ etm.2018.6904

\begin{abstract}
The aim of the study was to identify if there is a connection between the time of administration of alpha-blocker medication and cataract surgery complications. Furthermore, it was explored whether discontinuation of tamsulosin before cataract surgery influences the manifestations of intraoperative floppy iris syndrome in rats. An experimental study was conducted on 20 male Wistar rats aged 1.5-2 years (body mean weight $357 \mathrm{~g}$ ), which were divided into four equal groups: Group 1, under tamsulosin hydrochloride $0.4 \mathrm{mg}$ /day for 2 months; group 2, without any treatment in the first month, followed by tamsulosin; group 3, under tamsulosin for 1 month, followed by 1 month without any treatment; and group 4, control. The pupillary diameter was assessed before instillation of $0.5 \%$ tropicamide (mydriatic and cycloplegic agent), after 1 or $2 \mathrm{~h}$ from instillation and postoperatively. Furthermore, pupil constriction during surgery, the presence of floppy iris and prolapse of the iris following the main incision and during serum injection were also assessed. Other analyzed features included iris rupture, posterior capsule tear, vitreous loss, lens fragments in the vitreous, suprachoroidal bleeding and corneal haze. The iris dilator muscle thickness and the posterior epithelium was measured using light microscopy. In the control group, the largest pupillary diameters were indicated and in group 1, the smallest pupillary diameters were revealed. Statistically significant differences were indicated between group 1 and 2 and group 3 and 4. Floppy iris, iris prolapse in the main incision and during serum injection appeared at maximum frequency for group 1 with continuous treatment and low frequency for the control group. Lens fragments in the vitreous were
\end{abstract}

Correspondence to: Mrs. Raluca Maria Popescu, Department of Surgical Specialties, Faculty of General Medicine, 'Iuliu Haţieganu' University of Medicine and Pharmacy, 8 Victor Babes Street, 400012 Cluj-Napoca, Romania

E-mail: ralucaturc@yahoo.com

Key words: tamsulosin, alpha-blocker, cataract, benign prostatic hyperplasia, floppy iris present in the groups 1-3. Notably, intraoperative miosis had statistical relevance in group 1 and group 2 . To conclude, the present findings suggested there is a clear connection between the time of administration of the alpha-blocker medication and most cataract complications. The results indicated that discontinuation of tamsulosin for one month largely reduces the manifestations of intraoperative floppy iris syndrome in rats, which may suggest to change preoperative treatment for benign prostatic hypertrophy with another class of drugs that do not interact with the iris receptors.

\section{Introduction}

It is known that systemic alpha-blockers determine relaxation of the smooth muscle situated in the bladder and prostate, this class of drugs being used in the treatment of benign prostatic hypertrophy (BPH) (1). Most of the patients with prostate adenoma use tamsulosin hydrochloride, a sympathetic $\alpha$-1a antagonist drug, because it causes less postural hypotension (1). Alpha-blockers improve symptoms correlated to BPH, but also complicate cataract surgery by changing iris behaviour (1). The close relationship between intraoperative floppy iris syndrome (IFIS) and $\alpha$-blockers, especially tamsulosin, were reported thirteen years ago for the first time (1). IFIS has a frequency of $2 \%$ in cataract surgeries, being mostly caused by tamsulosin (2).

The reasons for causing IFIS and for its means of action in the treatment for BPH are the same: Tamsulosin causes relaxation of the smooth muscles in the bladder (the neck part of the bladder) and of the iris dilator muscle, alpha ${ }_{1 \mathrm{~A}}$ receptors being their targets (3). In some cases two weeks of treatment with tamsulosin were sufficient to cause IFIS (3). Tamsulosin is the most common drug implicated in IFIS, although it is not the only one which can determine this syndrome (3).

The iris is innervated by parasympathetic and sympathetic nerves and controls the size of the pupil and adjusts the amount of light received (4). The sympathetic regulates the contraction of iris dilator muscle through $\alpha_{1}$-adrenoceptors and the parasympathetic controls the contraction of sphincter muscle through muscarinic pathway in many species (4). Reverse transcription-polymerase chain reaction (RT-PCR) was used in studying the expression of mRNAs, structure that encodes proteins of $\alpha 1 \mathrm{a}, \alpha 1 \mathrm{~b}$ and $\alpha 1 \mathrm{~d}$-adrenoceptors (4). The $\alpha_{1 \mathrm{a}}$-adrenoceptor had 
the strongest expression, the $\alpha$ lb-adrenoceptor had a weak expression and $\alpha 1 \mathrm{~d}$-adrenoceptor had an undetectable expression (4). In the human prostate, $70 \%$ of the a1-receptors are $\alpha 1 \mathrm{a}$ subtype (5). Tamsulosin has a 20 times greater affinity for $\alpha 1$ a than $\alpha 1 b$ receptors according to in vitro data and animal studies ( $\alpha$ la being the dominant adrenoreceptor in the iris) (5).

The irony comes from the fact that while tamsulosin is treating $\mathrm{BPH}$, it multiplies the risk of preoperative, intraoperative and postoperative complications associated with another condition, cataracts (3). IFIS triad is composed of: Progressive and intraoperative miosis, iris prolapse and billowing of the iris (3). Some authors declare the incidence of IFIS among alpha-blocker treatment users between 33 and 78\%, the most common complication being iris trauma, followed by vitreous loss posterior and capsular tears (3).

This syndrome has a wide scale of severity described by other authors. Mild cases cause some iris billowing but dilate well, which can permit successful use of recommended techniques for controlling the unwanted intraoperative iris behavior (1). Severe cases can produce marked billowing of the iris, wild iris prolapse, marked miosis during surgery (1). Those require the use of iris hooks, expander or iris retractors in order to complete the surgery in safety conditions (1).

In this regard, our goal was to study albino Wistar rats, if there were the same changes in the rat iris dilator muscle as in rabbits, and if these changes were strictly related to the tamsulosin treatment time, but also to its preoperative discontinuation. We also chose the albino type to highlight the $\alpha 1 \mathrm{~A}$ receptor expression and quantify the importance of the iris dilatator muscle layer in IFIS manifestations.

\section{Materials and methods}

An experimental study was conducted on 20 Wistar male rats aged 1.5-2 years, body mean weight $357 \pm 26.63 \mathrm{~g}$, lasting 2 months, divided into four groups: Group 1, 5 rats under tamsulosin hydrochloride $0.4 \mathrm{mg} /$ day for 2 months; group 2 , 5 rats without any treatment in the first month, followed by tamsulosin hydrochloride $0,4 \mathrm{mg} /$ day for $1 \mathrm{month}$; group 3 , 5 rats under tamsulosin hydrochloride $0,4 \mathrm{mg} /$ day for 1 month, followed by 1 month without any treatment; group 4, 5 rats (control group), without any treatment. The calculation of the dose was performed according to the body surface area ratio man/rats; the median lethal dose LD50 and the accelerated rat metabolism were taken into account.

Adult male wistar rats were supplied by 'Iuliu Haţieganu' University of Medicine and Pharmacy (Cluj-Napoca, Romania), and accommodated into 'Establishment for breeding and use of laboratory animals' of University of Medicine and Pharmacy (UMF), Cluj-Napoca, Romania. Rats were housed in standard polypropylene cages, at optimum density and in standard laboratory conditions (temperature $25 \pm 1^{\circ} \mathrm{C}$, relative humidity $55 \pm 5 \%$, and $12 \mathrm{~h} \mathrm{light/dark} \mathrm{cycle).} \mathrm{The} \mathrm{rats} \mathrm{were} \mathrm{allowed} \mathrm{free}$ access to standard granular diet and water ad libitum.

All the procedures performed on laboratory animals, comply with the European Directive 22.09.2010/63/EU, and Romanian national low 43/2014 for protection of animals used for scientific purposes. This project was legally approved by the Comity for Bioethics of UMF (accord no. 336/31.08.2017) and the Veterinary Sanitary Direction and Food Safety.
Tamsulosin hydrochloride was administrated to each rat daily according to its group affiliation, before the first meal, as $0.4 \mathrm{mg}$ tamsulosin hydrochloride powder mixed in its food. The tamsulosin dose that we used was the one calculated by the anesthesiologist according to the accelerated metabolism of the rat: $1 \mathrm{mg}$ per kilogram body weight per day.

Surgery study in rats was done by the same specialist over two days using an adapted protocol for cataracts. The surgeon was not informed which group the animal belonged to before or during surgeries, the order being random and with a uniform distribution.

The anesthesia was performed with the combination of xylazine and ketamine. The doses administered were $5.8 \mathrm{mg}$ ketamine and $0.8 \mathrm{mg}$ xylazine per $100 \mathrm{~g}$ body weight, intraperitoneally. Except for the first rat, everyone responded to this dose. The animals did not wake up intraoperative. Euthanasia was induced by the single intraperitoneal administration of pentobarbital $40 \mathrm{mg} / 100 \mathrm{~g}$ body weight immediately after surgery. Successful euthanasia was confirmed after achieving a lack of heartbeat and respiratory movements, lack of reactivity to painful stimuli and rigor mortis.

The chosen protocol was the extraction of the lens by the phacoemulsification technique, but adapted to the given conditions. Most of the operator steps were followed, but without using ultrasound to remove the lens. Because of the small dimensions of the rat's lens, it was not possible to use the ultrasound probe used in the human patients. Thus, we performed the following steps in the operative protocol: Local anesthesia with oxybuprocaine hydrochloride, side-ports at 10 and $14 \mathrm{~h}$ with the 15 -degree $\mathrm{V}$ Lance knife, cannula introduction of physiological serum in the anterior chamber, corneo-scleral incision at the limbus level at 11-12 or temporal, coloration of the anterior capsule of the lens in some cases for better visualization, the capsulorhexis with 26-gauge twisted needle, hydrodissection and hydrodelineation of the nucleus by injecting the lens with serum, irrigation and aspiration of the remaining cortex, insertion of the serum and side-port sealing by injecting serum in the edges of the incisions.

Identification of the syndrome requires the presence of a triad of three clinical signs: i) Intraoperative progressive pupil constriction, ii) an iris that shows floppy as it billows while irrigation and aspiration is being performed in the anterior chamber, and iii) a clear tendency of the iris to prolapse first into the phacoemulsification and anter in the side-port incisions during surgery. This may or not be accompanied by a poorly dilated pupil before surgery.

Valid results for a modified iris behavior were obtained preoperative, intraoperative and postoperative. We noted the pupillary diameter at 9 a.m. the day of surgery (before instillation of $0,5 \%$ tropicamide-mydriatic and cycloplegic agent), after $1 \mathrm{~h}$ from instillation of tropicamide and after $2 \mathrm{~h}$ of instillation of tropicamide; the presence or absence of pupil constriction during surgery, floppy iris, the prolapse into the main incision or in side-ports, prolapse of the iris during serum injection, iris rupture, posterior capsule tear, vitreous loss, lens fragments in the vitreous, suprachoroidal bleeding, corneal haze. Postoperative we measured the pupil diameter and we calculated the total surgery time.

We noted cases of floppy iris as those situations where the iris was shaking and billowing. The prolapse in the main incision or in the sideport refers to the fact that the iris slides into the incisions 
during or even in the absence of surgical maneuvers. Prolapse of the iris during the injection of serum indicates slippage during injection through side ports. The iris rupture it's due to the iris atrophy under tamsulosin treatment and translates its friability. The posterior capsule can break more easily in the low visibility situation, stronger ultrasound intraoperative parameters, and intense surgical maneuvers when tamsulosin is used as a treatment. The posterior capsule of the lens can be easy broken in low visibility and high surgery parameters, intensive maneuvers, aggravating the operatory steps. Lens fragments may also appear in the vitreous by breaking the posterior capsule, complicating the surgery process. Due to excessive manipulation in these cases, suprachoroidal haemorrhage may occur, complication with small chances of recovery of postoperative vision. Corneal haze means a cloudy cornea, also being a result of high intraoperative parameters and a long total operative time.

We enucleated one eye for each group, including the control group, to perform histopathological examination of the iris.

Light microscopy. The eyes of rats from the experimental groups (G-I, G-II, G-III) and the control group were collected immediately after euthanasia, fixed in $10 \%$ phosphate-buffered formalin with $\mathrm{pH} 7.0(24 \mathrm{~h})$, processed through a tissue-specific protocol and embedded in paraffin wax. The 4 micrometer $(\mu \mathrm{m})$ sections were cut horizontally through the visual axis and routinely stained with hematoxylin and eosin (H\&E). The photomicrographs were taken using an Olympus SP 350 digital camera and Stream Basic imaging software (Olympus Corporation, Tokyo, Japan).

The thickness of the iris dilator muscle and the posterior epithelium was evaluated. The animals used in this study were albino Wistar rats; thus an assessment regarding the pigment granules in the posterior epithelium cells could not be performed.

Statistical analysis. All data are reported as the mean \pm SEM. The Gaussian distribution was checked by D'Agustino \& Person normality test. One-way analysis of variance ANOVA and followed by post hoc Bonferoni range test procedure was performed for pair-wise comparisons between the normally distributed data, while data with non-Gaussian distribution were analyzed by analysis (one-way) of variance Kruskal-Walis, followed by post test Dunns. Spearman's correlation was chosen, to assess the correlation between non normally distributed variables; the interpretation was made according to the Colton scale. Logistic regression model for the potential association between tamsulosin hydrochloride administration and various associated ocular pathology was analyzed by Fishers exact test followed by odds ratio. Statistical significance was at $95 \%$ confidence interval, $\mathrm{P}<0.05$. Statistical values and figures were obtained using GraphPad Prism version 5.0 for Windows, GraphPad Software (San Diego, CA, USA).

\section{Results}

We measured the pupillary diameters on the day of the surgery as follows: Before using tropicamide, one and two hours after instillation of tropicamide and finally at the end of the surgery. We also noted the moment when intraoperative miosis occurred (miosis was considered when the pupillary diameter was $\leq 4 \mathrm{~mm}$ ). The largest pupillary diameters were found in the control group (mean value $5.3 \mathrm{~mm}$ ), and in group 1 (mean value $4.7 \mathrm{~mm}$ ) with continuous treatment, the smallest pupil diameters. Statistically significant differences occurred when comparing group 2/group $3(\mathrm{P}<0.05)$ and group 2/control group $(\mathrm{P}<0.05)$ before using tropicamide and before the surgery (Fig. 1A). One hour after the tropicamide infiltration, statistically significant P-values were found for the following comparative situations: Group 2/group 4 and group 1/group 4. In both cases $\mathrm{P}<0.01$. (Fig. 1B). After $2 \mathrm{~h}$ of tropicamide administration, the $\mathrm{P}$-value was $<0.05$ when comparing group 2 with group 4 and group 2 with group 3 or group 1 with group 3 . The P-value was $<0.01$ when comparing groups 1 with 4 . (Fig. 1C). At the end of the surgery there were statistically significant differences between the four groups as follows: $\mathrm{P}<0.5$ in case of comparison of group 2 with group 3, group 2 with group 4; $\mathrm{P}<0.01$ while comparing group 1 to group 3 and $\mathrm{P}<0.001$ while comparing group 1 (mean value $2.9 \mathrm{~mm}$ ) to group 4 (mean value $5.6 \mathrm{~mm}$ ) (Fig. 1D and Fig. 2A-D). The total duration of the intervention post-anesthesia (measured in min) was longer for group 1 and 2 compared to control group 4 $(\mathrm{P}<0.05$; Fig. 3$)$. The total amount of intraoperative serum (measured in $\mathrm{ml}$ ) was not statistically significantly higher in group 1 with continuous treatment (Fig. 4).

Floppy iris occurred at peak frequency for group 1 with continuous treatment (Fig. 5A and B) and with zero frequency in the control group. Group 2 (odds ratio, 30.33; confidence interval 95\%, 3.002-1698; $\mathrm{P}=0.01$ ), treated with tamsulosin in the last part of the study followed group 1 regarding the presence of floppy iris (odds ratio, 71.4; confidentiality interval 95\%, 1.391-661.3; $\mathrm{P}<0.001$ ); group 3 (odds ratio, 6.176; confidentiality interval 95\%, 0.2597-146.9; $\mathrm{P}>0.05$ ) was next, followed by the control group with zero cases (Table I).

Iris prolapse in the main incision appeared with maximum frequency for group 1 with continuous treatment (Fig. 5B) and low frequency (1 case) for the control group. Group 2 (odds ratio, 81; confidentiality interval 95 , 4.358-1506; $\mathrm{P}=0.001$ ), undergoing treatment in the last part of the study, followed group 1 (odds ratio, 133; confidence interval 95\%, 4.810-3677; $\mathrm{P}<0.001)$. Group 3 recorded as follows: Odds ratio, 3.857; confidence interval 95\%, 0.3263-45.60; P=0.5 (Table I).

Iris prolapse during serum injection occurred with the highest frequency for group 1 , the one with continuous treatment (Fig. 5B-D) and low frequency (1 case) in the control group. Group 2 (odds ratio, 36; confidence interval 95\%, 2,719-476.6; $\mathrm{P}=0.005$ ), followed group 1 (odds ratio, 81; confidence interval 95\%, 4.358-1506; $\mathrm{P}<0.01$ ); in group 3 (odds ratio, 9; confidence interval 95\%, 0.8084-100.2; P>0.05) few cases were reported (Table I).

The rupture of the posterior capsule (posterior capsule tear) had a null intraoperative presence in the control group 4 but met among the other three groups as an intraoperative complication: Group 3 (odds ratio 1, confidence interval 95\%, 0.1199-91.68; $\mathrm{P}=1$ ) group 2 (odds ratio, 6.17, confidence interval 95\%, 0.2597-146.9'; $\mathrm{P}=0.47$ ) group 1 (odds ratio, 14.54; confidentiality interval 95\%. 0.6669-316.9; $\mathrm{P}=0.08$ ) (Table I).

Lens fragments in the vitreous were present in the first three groups (Fig. 6A) and in the 4th group no such intraoperative complication was recorded. The complication had the following distribution: Group 3 (odds ratio 1, 
A

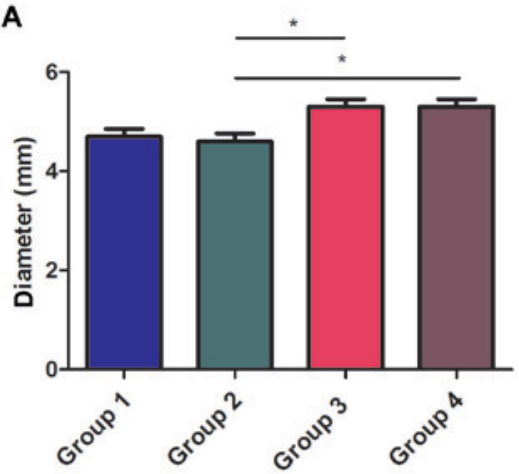

C

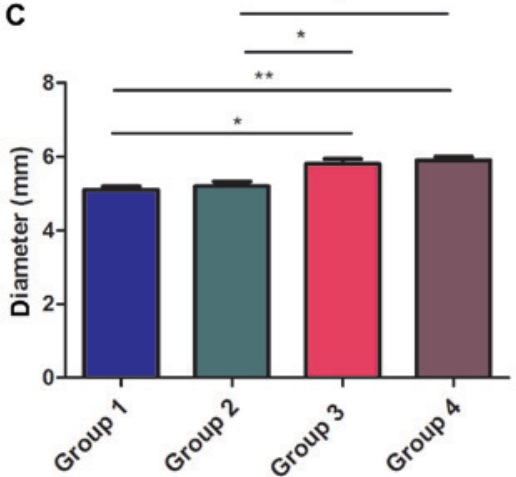

B

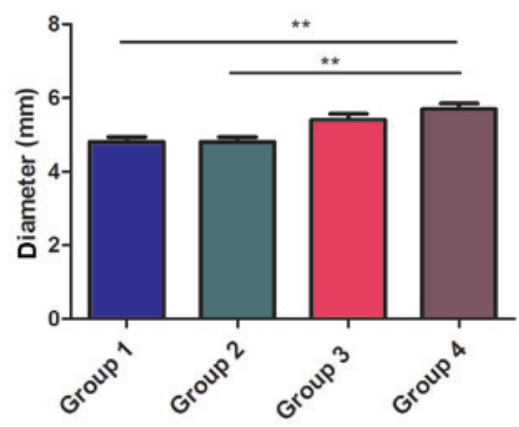

D

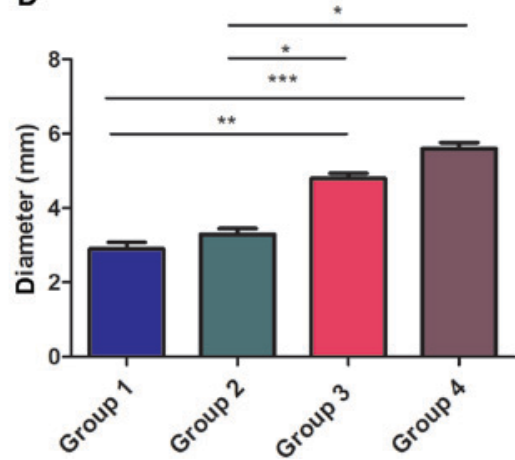

Figure 1. Pupillary diameter: (A) Before the first tropicamide instillation; (B) after one hour from the first tropicamide instillation; (C) after two $\mathrm{h}$ from the first tropicamide instillation; (D) at the end of the surgery (mm). Data were presented as the mean \pm SEM (10 samples/group). ${ }^{*} \mathrm{P}<0.05,{ }^{* *} \mathrm{P}<0.01$ and ${ }^{* * * *} \mathrm{P}<0.001$ (Kruskal-Wallis with Dunn's post hoc test).

confidence interval 95\%, 0.05380-18.59; $\mathrm{P}=1$ ), group 2 (odds ratio, 3.8; confidence interval 95\%, 0.3263-45.60; $\mathrm{P}=0.58$ ) group 1 (odds ratio, 9; confidence interval 95\%, 0.8084-100.2; $\mathrm{P}=0.1409$ (Table I).

Suprachoroidal bleeding (haemorrhage) was installed in the following cases: Group 1 (odds ratio, 6.176; confidence interval 95\%, 0.2597-146.9), group 2 (odds ratio, 3.31; confidence interval 95\%, 0.1199-91.68), group 3 (odds ratio, 1 ; confidence interval 95\%, 0.05459-18.32) (Fig. 6B and F); group 4 not installed at all. The P-values were $>0.05$, not statistically significant (Table I).

The corneal haze complicated the cataract surgery as follows: Group 1 (odds ratio, 9,000; confidence interval 95\%, 0.8084-100.2), group 2 (odds ratio, 3.857; confidence interval 95\%, 0.3263-45.60), group 3 (odds ratio, 1,000; confidence interval $\left.95 \%, 0.05380-18.59^{\prime}\right)$ and group 4 with only one case (Table I).

The iris rupture was not present in the control group, but it appeared with decreasing frequency in the following groups: Group 1 (odds ratio, 9.000; confidence interval 95\%, 0.8084-100.2), group 2 (odds ratio, 3.857; confidence interval 95\%, 0.3263-45.60), group 3 (odds ratio, 3.316; confidence interval 95\%, 0.1199-91.68) (Fig. 6C). The P-values were all $>0.05$, not statistically significant (Table I).

Loss of vitreous was recorded as follows: Group 1 (odds ratio, 6.000; confidence interval 95\%, 0.5318-67.69), group 2 (odds ratio, 2.250; confidence interval 95\%, 0.1700-29.79), group 3 (odds ratio, 0.3016; confidence interval 95\%, 0.01091-8.338) (Fig. 6D). The P-values were all $>0.05$, not statistically significant (Table I).

Intraoperative miosis had statistical relevance in group 1 and group 2. Group 1 had a odds ratio of 441.0 (confidence interval
95\%, 7.972-24400; $\mathrm{P}<0.0001$ ), group 2 had a odds ratio of 441.0 (confidence interval 95\%, 7.972-24400; $\mathrm{P}<0.0001$ ) and group 3 odds ratio, 6.176 (confidence interval 95\%, 0.2597-146.9; $\mathrm{P}>0.05$ ) (Fig. 6C-F) (Table I).

The thickness of the iris dilator muscle and the posterior epithelium were evaluated (Table II). The measurements of the thickness of the iris dilator muscle changed depending on the duration of the alfa-blocker treatment. In other words, the 4th control group recorded a thickness of $2.85 \mu \mathrm{m}$, the group 3 had a smaller thickness $(2.76 \mu \mathrm{m})$, followed by group 2 with $1.59 \mu \mathrm{m}$ and group 1 which had the smallest thickness of $0.97 \mu \mathrm{m}$. A significant difference can be seen between the control group 4 and the group 1, both in terms of the size of the iridium dilator muscle layer and in terms of the thickness of the posterior epithelium (group 1 having a thickness of $4.83 \mu \mathrm{m}$, while group 4 of $10.63 \mu \mathrm{m}$ ) (Figs. 7 and 8).

In the microscopic images below, we can see the thinning of the iris dilator muscle layer, as the duration of treatment with tamsulosin increases. The atrophy of the iris dilator muscle is evident in the picture bellow, where the iris section of an eyeball belonging to group 1, with continuous treatment, is illustrated (Fig. 9A). The muscle atrophy is also seen in group 2 (Fig. 9B) with treatment over the last month but is not as obvious as in the previous case. Groups 3 and 4 (control) do not show any major changes to each other, group 4 being the reference in our case (Fig. 9C and D).

\section{Discussion}

The distribution on the four groups helped us to highlighting the effects of tamsulosin hydrochloride, which is a prostate 

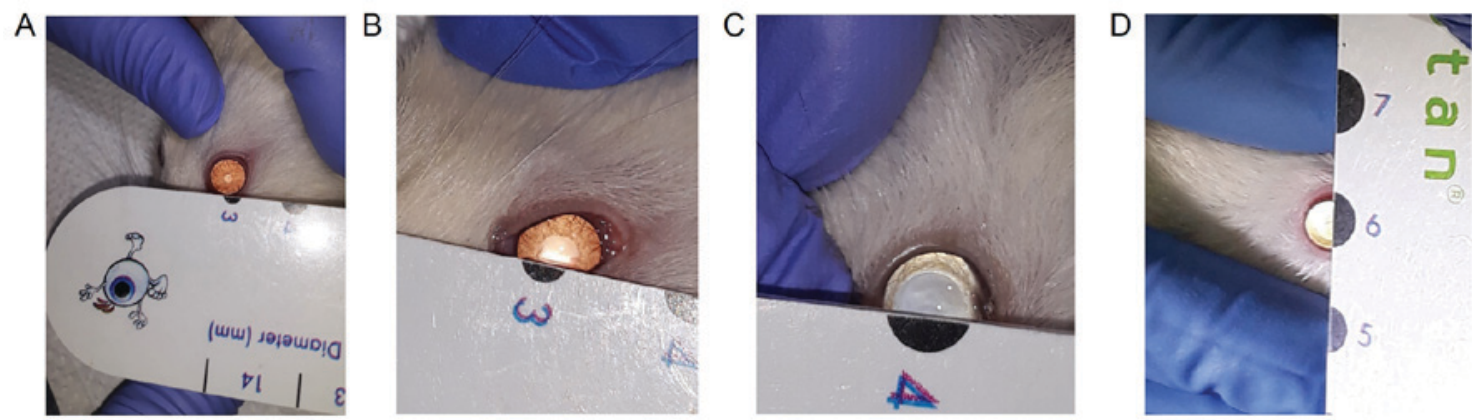

Figure 2. Pupillary measurements immediately postoperative: (A) excipient from group 1; (B) excipient from group 2; (C) excipient from group 3; and (D) excipient from group 4.

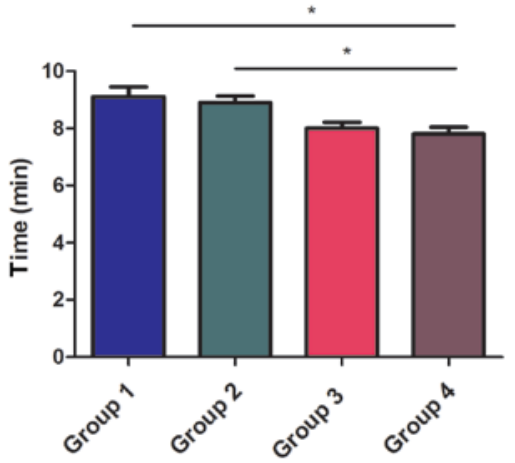

Figure 3. Post-anesthesia duration of the procedure (minutes). Data are presented as the mean \pm SEM (10 samples/group). ${ }^{*} \mathrm{P}<0.05$ (Kruskal-Wallis with Dunn's post hoc test).

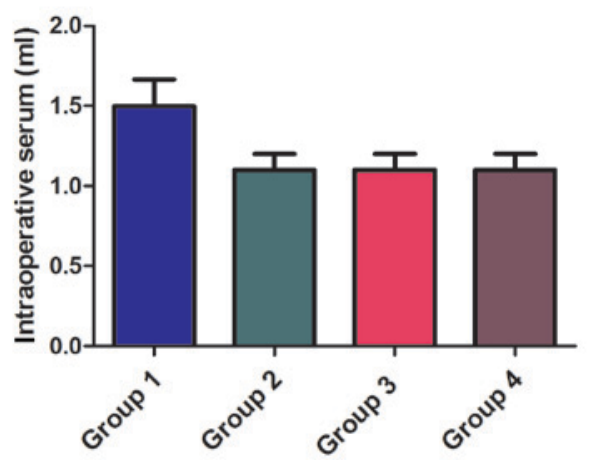

Figure 4 . The total amount of intraoperative serum $(\mathrm{ml})$. Data are presented as the mean \pm SEM (10 samples/group) (two-way analysis with Bonferroni's post hoc test).
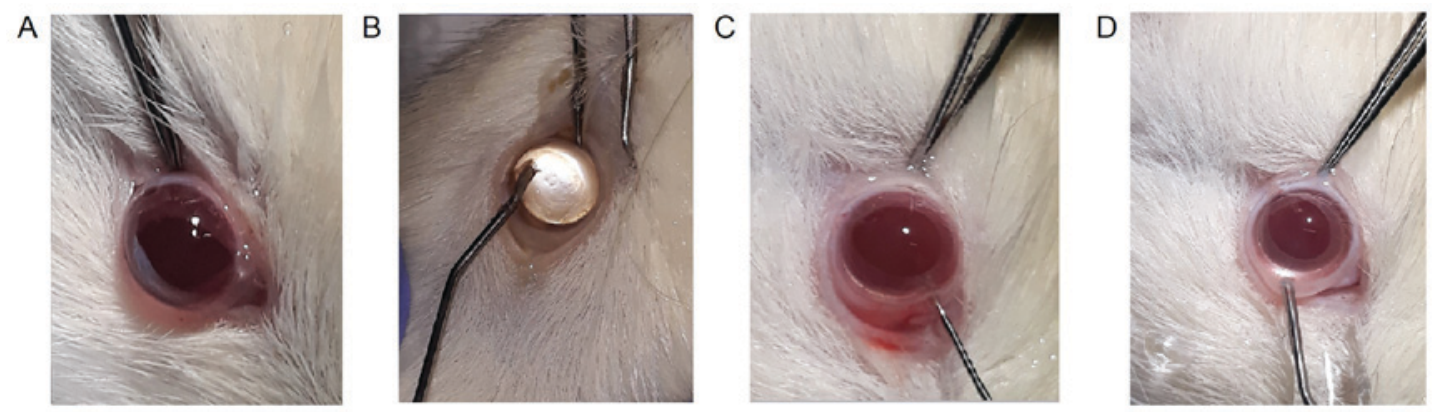

Figure 5. (A) Floppy iris and iris prolapse in the main incision (excipient from group 1); (B) floppy iris and iris prolapse during serum injection (excipient from group 1); (C) iris before serum injection-the pupil is in shape (excipient from group 1); and (D) floppy iris and iris prolapse during serum injection (excipient from group 1).

adenoma medication, at the iris level. In other words, group 1, with continuous medication in the two months, had the most preoperative, intraoperative and postoperative complications, followed by group 2 , receiving medication only in the last month, which was followed by group 3 with medication in the first month and a month break after; group 1 did not have any specific manifestation, being the control one. One can see a similarity between groups 1 and 2 (those with continuous treatment, respectively with treatment in the last one) and between groups 3 and 4 (treatment group in the first month followed by one month break and the control group).

In terms of pupil diameter in the control group (group 4), we found the highest pupillary diameters, and in the case of group 1, with continuous treatment, the smallest pupil diameters, as we expected. The greater statistically significant difference was between the continuous treatment group and the control group. In other words, we found in all the situations the lowest pupillary diameter values in the groups 1 and 2 , while groups 3 and 4 showed elevated values.

Intraoperatory floppy iris syndrome was characterized by Chang et al for the first time as a flaccid iris (5). Flutter moves were recorded during the procedure of phacoemulsification, running at normal current levels concerning the circulating fluid, iris prolapse in the main incision and through the side-ports, but also intraoperative miosis $(6,7)$. We discovered floppy iris in $80 \%$ in the first group, the one with continous treatment. Group $2(\mathrm{P}=0.1)$, the one with treatment in the last part of the study, followed group $1(\mathrm{P}<0.001)$ as frequency, and 
Table I. Logistic regression model for the potential association between tamsulosin hydrochloride administration and various associated ocular pathology (Fishers exact test followed by odds ratio).

\begin{tabular}{|c|c|c|c|}
\hline Variable & Odds ratio & $95 \%$ Confidence interval & P-value \\
\hline \multicolumn{4}{|l|}{ Floppy iris } \\
\hline Group 1 & 71.40 & $3.002-1698$ & 0.0007 \\
\hline Group 2 & 30.33 & $1.391-661.3$ & 0.0108 \\
\hline Group 3 & 6.176 & $0.2597-146.9$ & 0.4737 \\
\hline Group 4 & 1.00 & Reference category & \\
\hline \multicolumn{4}{|c|}{ Iris prolapse in main incision } \\
\hline Group 1 & 133.0 & $4.810-3677$ & 0.0001 \\
\hline Group 2 & 81.00 & $4.358-1506$ & 0.0011 \\
\hline Group 3 & 3.857 & $0.3263-45.60$ & 0.5820 \\
\hline Group 4 & 1.00 & Reference category & \\
\hline \multicolumn{4}{|c|}{ Iris prolapse while serum injection } \\
\hline Group 1 & 81.00 & $4.358-1506$ & 0.0011 \\
\hline Group 2 & 36.00 & $2.719-476.6$ & 0.0055 \\
\hline Group 3 & 9.000 & $0.8084-100.2$ & 0.1409 \\
\hline Group 4 & 1.00 & Reference category & \\
\hline \multicolumn{4}{|c|}{ Lens fragments in vitreous } \\
\hline Group 1 & 9.000 & $0.8084-100.2$ & 0.1409 \\
\hline Group 2 & 3.857 & $0.3263-45.60$ & 0.5820 \\
\hline Group 3 & 1.000 & $0.05380-18.59$ & 0.9999 \\
\hline Group 4 & 1.00 & Reference category & \\
\hline \multicolumn{4}{|c|}{ Posterior capsule tear } \\
\hline Group 1 & 14.54 & $0.6669-316.9$ & 0.0867 \\
\hline Group 2 & 6.176 & $0.2597-146.9$ & 0.4737 \\
\hline Group 3 & 3.316 & $0.1199-91.68$ & 0.9999 \\
\hline Group 4 & 1.00 & Reference category & \\
\hline \multicolumn{4}{|c|}{ Suprachoroidal haemorrhage } \\
\hline Group 1 & 6.176 & $0.2597-146.9$ & 0.4737 \\
\hline Group 2 & 3.316 & $0.1199-91.68$ & 0.9999 \\
\hline Group 3 & 1.000 & $0.05459-18.32$ & 0.9999 \\
\hline Group 4 & 1.00 & Reference category & \\
\hline \multicolumn{4}{|c|}{ Corneal haze } \\
\hline Group 1 & 9.000 & $0.8084-100.2$ & 0.1409 \\
\hline Group 2 & 3.857 & $0.3263-45.60$ & 0.5820 \\
\hline Group 3 & 1.000 & $0.05380-18.59$ & 0.9999 \\
\hline Group 4 & 1.00 & Reference category & \\
\hline \multicolumn{4}{|l|}{ Iris rupture } \\
\hline Group 1 & 9.000 & $0.8084-100.2$ & 0.1409 \\
\hline Group 2 & 3.857 & $0.3263-45.60$ & 0.5820 \\
\hline Group 3 & 3.316 & 0.1199-91.68 & 0.9999 \\
\hline Group 4 & 1.00 & Reference category & \\
\hline \multicolumn{4}{|c|}{ Loss of vitreous } \\
\hline Group 1 & 6.000 & $0.5318-67.69$ & 0.3034 \\
\hline Group 2 & 2.250 & $0.1700-29.79$ & 0.9999 \\
\hline Group 3 & 0.3016 & $0.01091-8.338$ & 0.9999 \\
\hline Group 4 & 1.00 & Reference category & \\
\hline \multicolumn{4}{|c|}{ Intraoperative miosis } \\
\hline Group 1 & 441.0 & $7.972-24400$ & $\mathrm{P}<0.0001$ \\
\hline Group 2 & 441.0 & $7.972-24400$ & $\mathrm{P}<0.0001$ \\
\hline Group 3 & 6.176 & $0.2597-146.9$ & 0.4737 \\
\hline Group 4 & 1.00 & Reference category & \\
\hline
\end{tabular}


A

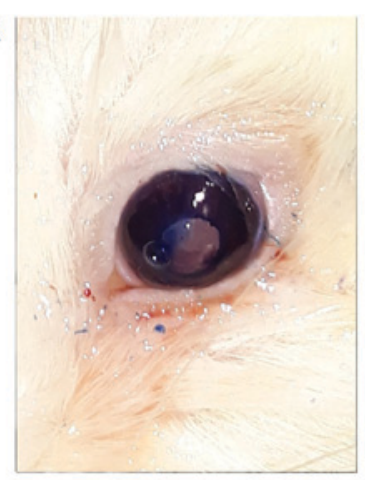

D

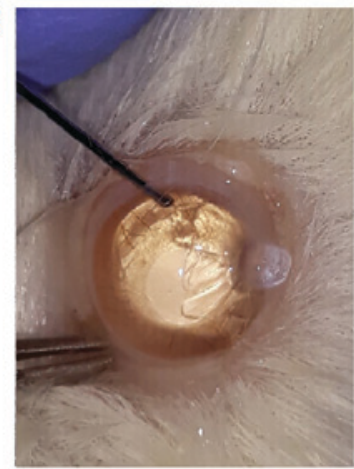

B

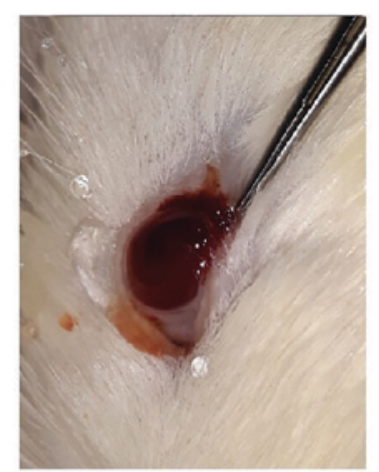

E

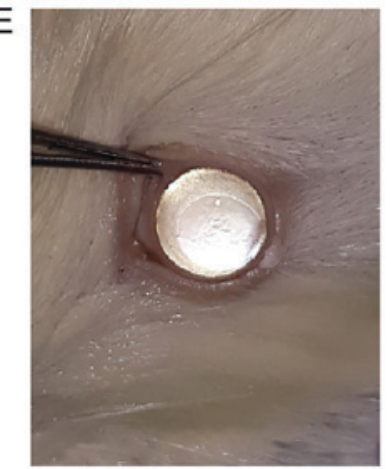

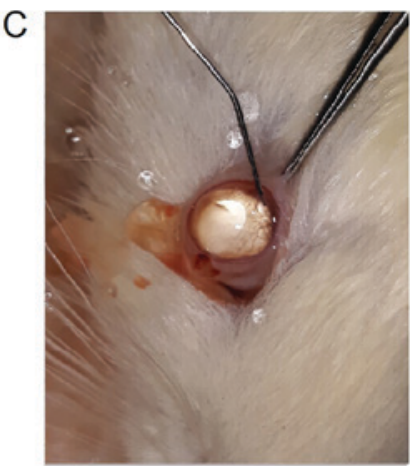

$\mathrm{F}$

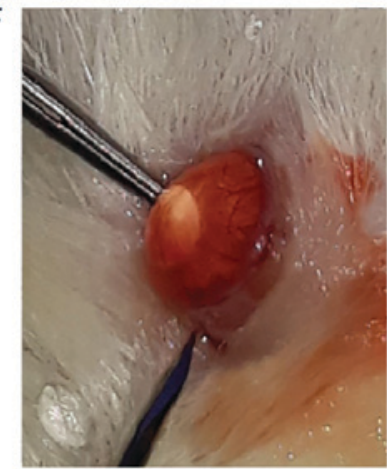

Figure 6. (A) Lens subluxation in the vitreous; (B) anterior chamber and suprachoroidal bleeding; (C) iris rupture, intraoperative miosis and iris prolapse; (D) vitreous loss and intraoperative miosis; (E) intraoperative miosis and iris in the main incision; and (F) intraoperative miosis, anterior chamber and suprachoroidal bleeding.

in group 3 there were the fewest cases. We found iris prolapse through the main incision in $100 \%$ cases during surgery and $90 \%$ during serum introduction in the control group. In order to minimize this effect, authors recommend using viscoelastic substance, meaning substance with big molecular weight and a correctly performed incision $(6,7)$. Predisposing factors regarding the iris prolapse were revealed: Improper iris configuration, low anterior chamber depth, and unnormal architecture or position made by the surgeon of the corneal tunnel (8).

In one study on men operated for cataracts undergoing treatment for BPH, 23\% showed IFIS, but only the ones treated with tamsulosin (8). The severity and of course the incidence of IFIS was highly reduced after introducing a protocol, including iris retractors, minimal phaco parameters, intracamerular phenylephrine and high quantity of vascoelastic material (8). Bimanual phacoemulsification can be somethimes sufficient, without any need to use something else (6). Chang's suggestion of using a mantainer and a chopper helps keeping this way the circulatory irrigation in another position, anterior to the iris, consequently reducing the behavior of the iris (5). Storr-Paulsen finds in other words the same manifestations: significant inraoperative miosis, less preoperative dilatation and greater endothelial cell loss in comparision with patients without treatment for BPH, despite the recommended precautions taken (9). Other studies reccomends sub-Tenon lidocaine injection, which may reduce the incidence of floppy iris in comparison with intra-cameral lidocaine (10). Intracameral phenylephrine can reverse IFIS, causing iris rigidity, the pupil returning this way to its preoperative size (11). Tamsulosin performs its long action by a constant blockade at the level of the iris receptors, leading to an diffuse dilator muscle atrophy $(1,8)$. This may explain the poor preoperative dilation and the floppy iris phenomenon $(6,7)$.

The posterior capsule tear had a null intraoperative presence in control group 4 but met among the other 3 groups as an intraoperative complication, but with no statistical significance. We had five cases of posterior capsule rupture (50\%) with vitreous loss in the first group, which would had required anterior vitrectomy. Other authors have had a $12 \%$ frequency of this complication in cases with IFIS (7). Lens fragments in the vitreous were present in the first 3 groups, but in the 4th group, no such intraoperative complication was recorded. The iris rupture was not present in the control group, but it appeared with descending frequency in the following groups: 1, 2, 3 in this order. The P-values were not statistically significant. The loss of vitreous was recorded at high frequency in group 1 as we expected. Intraoperative miosis was installed with statistical relevance in group 1 and $2, \mathrm{P}<0.0001$.

Our goal was to study Wistar albino rats, more exactly if these changes are strictly related to tamsulosin treatment time and also to its preoperative discontinuation. Nakamura was the one who revealed, at the level of iris dilator smooth muscle, the existence of the alphal receptor in rabbits (4). We found out from our study that there is a clear connection between the time of administration of the alpha-blocker medication and most of the operative complications, especially in the case of intraoperative miosis, floppy iris and iris prolapse. There was a close similarity between the two groups (1-with continuous treatment and 2-with treatment in the last month) with minimal statistical differences and also a similarity between the other two groups (3-with treatment one month and last month pause and 4-the control one). From the last statement, 
Table II. The thickness of the iris dilator muscle and the posterior epithelium $(\mu \mathrm{m})$.

\begin{tabular}{lc}
\hline Component evaluated & Length $(\mu \mathrm{m})$ \\
\hline Posterior epithelium group 1 & 4.83 \\
Iris dilator muscle group 1 & 0.97 \\
Posterior epithelium group 2 & 10.97 \\
Iris dilator muscle group 2 & 1.59 \\
Posterior epithelium group 3 & 11.52 \\
Iris dilator muscle group 3 & 2.76 \\
Posterior epithelium control group 4 & 10.63 \\
Iris dilator muscle control group 4 & 2.83 \\
\hline
\end{tabular}

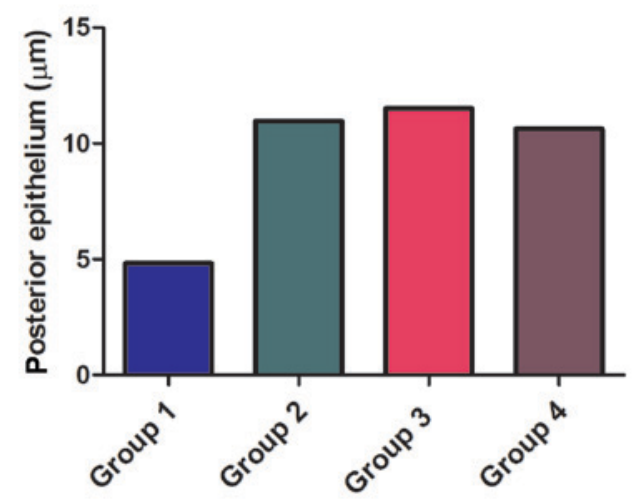

Figure 7. The thickness of the posterior epithelium $(\mu \mathrm{m})$.

we can emphasize the importance of stopping treatment with tamsulosin prior to cataract surgery in Wistar rats, with one month being optimal for good surgical outcomes.

The reason why we chose the albino type was to highlight the $\alpha 1 \mathrm{~A}$ receptor expression and to strictly quantify the importance of the iris dilatator muscle layer in the IFIS manifestations, considering that this type has no pigmentary granules in the posterior epithelial layer. We demonstrated the thinning of the posterior epithelium, but especially the thinning of the iridium dilator muscle, even its evident atrophy in group 1 and 2. All of these histological changes had echoes in the intraoperative iris floppy syndrome, as the literature had revealed: Melanin at the level of the posterior epithelial layer influence IFIS manifestations (12). We have expanded our research on the Wistar rats, as experiments have so far been carried out only on albino rabbits. Researchers also analyzed using electron microscopy (EM) on pigmented rabbit the structure of the iris that received tamsulosin vs. groups without treatment (12). They noted clumping of pigment granules, irregularities in the size and also thinning (atrophy) of the dilator muscle (12). There was a more lobular and irregular nuclei of the pigment epithelium and dilator muscle, quite similar to the human specimens (12). In this study, the authors suggests, as presented before, that the ala antagonist high affinity for melanin, which was found in the epithelial cells, may contribute to dilator muscle atrophy (12). For stronger evidence regarding their hypothesis, EM analyses of albino and pigmented rabbit eyes (under $\alpha 1 \mathrm{a}$

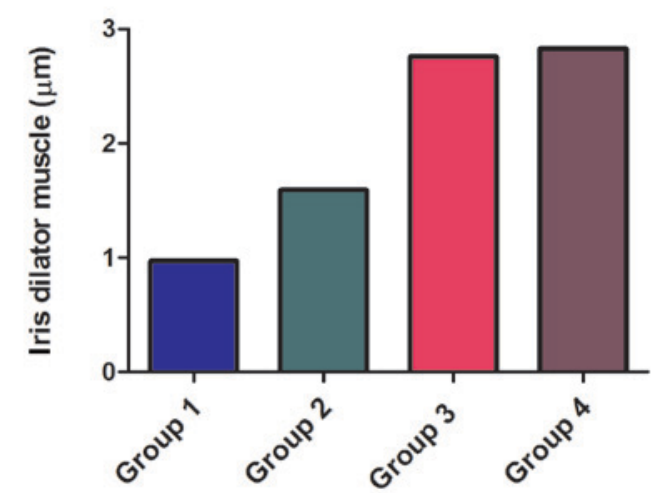

Figure 8 . The thickness of the iris dilator muscle $(\mu \mathrm{m})$.

antagonists treatment) could be recommended (12). There was no association between IFIS and the iris color in a prospective clinical study, suggesting that brown-eyed patients are not more susceptible to the $\alpha 1$ antagonist than blue-eyed men. Maybe a prospective study using individuals and correlating administration of tamsulosin, iris color and severity of IFIS can help to demonstrate the quantity of contribution regarding treatment-melanin interactions in IFIS (12).

A study used iris dilator muscles from rabbits and measured the tension at the iris level in response to a substance named phenylephrine with and without an $\alpha 1$ agonist (13). They analyzed the concentration curve as a response to phenylephrine in albino Japanese rabbits and Dutch pigmented rabbits (13). Compared to albino iris, phenylephrine had a much higher binding affinity for pigmented iris to the $\alpha 1$ receptor (13). That may suggest that the melanin pigment allows increased binding concerning the $\alpha 1$ receptor (13). In these sense, they concluded that the albino and pigmented rabbit iris are in other words identical except for the melanin presence (13). It could be interesting, in our case, the comparison on this matter of pigmented rats with Wistar rats, to see if there is more obvious effects of tamsulosin in cataract surgery in pigmented rats.

The incidence of IFIS according to Graefe $(1,9 \%)$ was significantly higher amongst tamsulosin and doxazosin users and suggested that all men under $\alpha$ lantagonists treatment should be identified before surgery and have alternative treatment and surgery techniques made by an experimented surgeon (14). Clinical and laboratory evidence suggest that there is a long-term anatomic and structural changes after the use of these drugs, which explain the IFIS persistence months after stopping the $\alpha$-blockers treatment (15).

In our study, in contrast to what we discussed before regarding the long-term effects of tamsulosin upon the iris, we have shown that discontinuation of tamsulosin for one month (group 3) largely cancels the manifestations of IFIS in rats. This may suggest a closer collaboration between the urologist and the ophthalmologist, with the urologist's suggestion to change treatment for BPH with another class of drugs that do not interact with the iris receptors. We are waiting for a definitive study which may delineate IFIS pathogenesis and its connection to drugs and diseases (15). Discontinuing $\alpha$-blockers prior to the patient's cataract surgery without drug replacement may not be in the favor of the patient (15). 
A

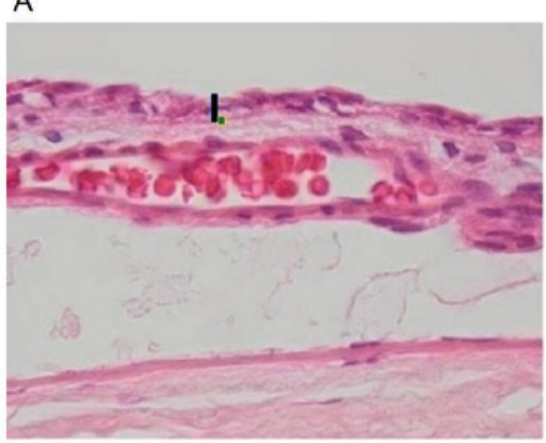

C

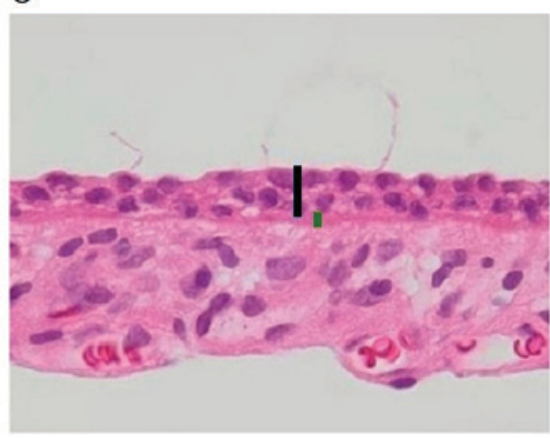

B

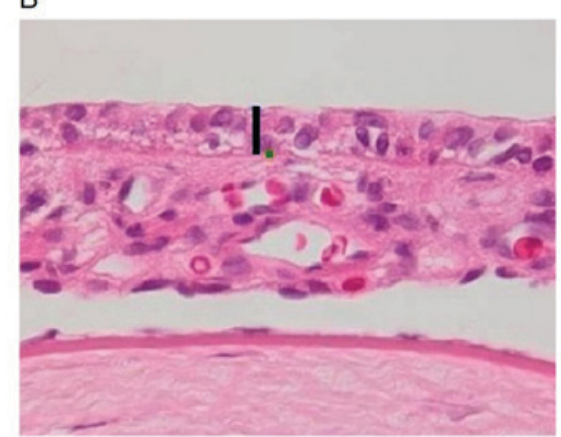

D

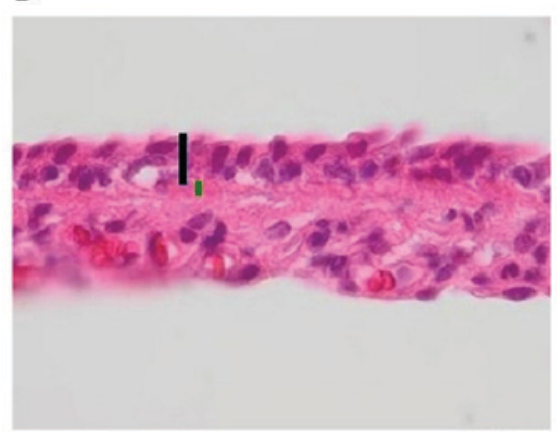

Figure 9. Histological images representing the posterior epithelium and the iris dilator muscle layer. (A) Iris: Black bar, posterior epithelium; green bar, iris dilator muscle (group 1). (B) Iris: Black bar, posterior epithelium; green bar, iris dilator muscle (group 2). (C) Iris: Black bar, posterior epithelium; green bar, iris dilator muscle (group 3). (D) Iris: Black bar, posterior epithelium; green bar, iris dilator muscle (control group 4). Histological analysis, hematoxylin and eosin stain; scale bar, $10 \mu \mathrm{m}$.

The other major group of drugs used to treat BPH seems that does not cause IFIS (16). Finasteride, a member of the 5 -alpha reductase inhibitors, is a drug typically used for BPH as first line treatment and androgenic alopecia, but is also associated sometimes with cataracts (17). Several studies have been reported, suggesting the existence of a link between IFIS and some other drugs, like: Finasteride, mianserin, antipsychotics (18-20). Likewise, another relationship between IFIS and other drugs was reported; for example, between IFIS and these drugs: chlorpromazine, aspirin, losartan, metformin (21,22); donepezil and quetiapine, imipramine, risperidone (23-26). Duloxetine and warfarin were also reported $(27,28)$.

There have been situations in which men with BPH have interrupted tamsulosin for several months, years and still have the classic triad of the syndrome and severe IFIS $(29,30)$. In other words, we cannot rely on stopping the treatment to cancel the IFIS symptoms (30). The duration of tamsulosin intake is not linked to the severity of the condition (15).

As a conclusion, the fact that the symptoms of intraoperative syndrome disappeared after stopping treatment with alpha-blockers a month before surgery in rats (on the contrary in humans stopping treatment more time before does not prevent the appearance of IFIS signs) leads us to the importance of the melalnin granules (the lack of melanin granules in albino rats, respectively their presence in humans) and the accelerated metabolism of rats. As we said above, there is clear evidence that there is a statistically significant difference between pigmented and albino rabbits, demonstrated by others, in terms of histological changes in the iris after alfa-blocker treatment, as well as between control group and group undergoing continuous treatment, demonstrated in our study on albino rats.

\section{Acknowledgements}

Not applicable.

\section{Funding}

No funding was received.

\section{Availability of data and materials}

The datasets used and analyzed during the current study are available from the corresponding author on reasonable request.

\section{Authors' contributions}

RMP designed the study, wrote the article, analyzed and interpreted the data, performed surgery on rats and was involved in drug administration. $\mathrm{CO}$ contributed in analysis of the data and was involved in drafting the manuscript. BS performed the statistical analysis and made the charts. MN and MT performed the histological examination of the enucleated eyes. IM performed the anesthesia and drug dose calculation. SB was responsible for the accommodation, feeding and drug administration for the animals and captured the intraoperative images. $\mathrm{CN}$ assisted in analyzing and interpreting the data, contributed to the study design and gave final approval of the version to be published. IC assisted in analyzing and interpreting the data, revised the manuscript 
critically for important intellectual content and gave final approval of the version to be published. All authors read and approved the final manuscript.

\section{Ethics approval and consent to participate}

All the procedures performed on laboratory animals comply with the European Directive 22.09.2010/63/EU and Romanian national law 43/2014 for protection of animals used for scientific purposes. The project was approved by the Comity for Bioethics of UMF (accord. no. 336/31.08.2017) and the Veterinary Sanitary Direction and Food Safety.

\section{Patient consent for publication}

Not applicable.

\section{Competing interests}

The authors declare that they have no competing interests.

\section{References}

1. Flach AJ: Intraoperative floppy iris syndrome: Pathophysiology, prevention, and treatment. Trans Am Ophthalmol Soc 107: 234-239, 2009

2. Chang DF and Campbell JR: Intraoperative floppy iris syndrome associated with tamsulosin. J Cataract Refract Surg 31: 664-673, 2005.

3. Janjua S and Cremers S: Managing intraoperative floppy iris syndrome. Cataract removal surgery and intraocular lens implantation: HS-100: 3-5, 2016.

4. Nakamura S, Tanigukhit T, Suzuki F, Akagi Y and Muramatsu I: Evaluation of alfa1 adrenoreceptors in the rabbit iris: Pharamcological caracterisation and expression of m RNA. Br J Pharmacol 127: 1367-1374, 1999.

5. Chang DF, Braga-Mele R, Mamalis N, Masket S, Miller MK, Nichamin LD, Packard RB and Packer M; ASCRS Cataract Clinical Committee: ASCRS white paper: Clinical review of intraoperative floppy-iris syndrome. J Cataract Refract Surg 34 2153-2162, 2008.

6. Nicula C, Nicula D and Popescu R: Intraoperative floppy iris syndrome-a prospective study. Oftalmologia 57: 38-44, 2013 (In Romanian).

7. Mamalis N: Importance of pupil dilation for cataract surgery. J Cataract Refract Surg 43: 583-584, 2017.

8. Chiseliţă D, Cantemir A, Epifanov L, Huţuleac A and Irod A: Management of intraoperative floppy iris syndrome. Oftalmologia 56: 69-76, 2012 (In Romanian).

9. Storr-Paulsen A, Jørgensen JS, Norregaard JC and Thulesen J: Corneal endothelial cell changes after cataract surgery in patients on systemicsympathetic $\alpha$-1a antagonist medication (tamsulosin). Acta Ophthalmol 92: 359-363, 2014.

10. Klysik A and Korzycka D. Sub-tenon injection of 2\% lidocaine prevents intra-operative floppy iris syndrome (IFIS) in male patients taking oral $\alpha$-adrenergic antagonists. Acta Ophthalmol 92: 535-540, 2014

11. Lorente R, de Rojas V, Vázquez de Parga P, Moreno C, Varela J, Landaluce ML, Méndez J and Lorente B: Intracameral phenylephrine $1.5 \%$ for prophylaxis against intraoperative floppy iris syndrome: Prospective, randomized fellow eye study. Ophthalmology 119: 2053-2058, 2012.
12. Chao DL, Modi YS, Lee B, Sridhar J, Kuriyan AE and Gedde SJ: Review of effects of tamsulosin and silodosin on isolated albino and pigmented rabbit iris dilators: Possible mechanism of intraoperative floppy-iris syndrome. Eye World ASCRS Publication, 2012.

13. Masket S and Chang DF: Intraoperative Floppy Iris Syndrome A systemic approach. J Cataract Refract Surg Today 10: 68-73, 2010.

14. Haridas A, Syrimi M, Al-Ahmar B and Hingorani M: Intraoperative floppy iris syndrome (IFIS) in patients receiving tamsulosin or doxazosin-a UK-based comparison of incidence and complication rates. Graefes Arch Clin Exp Ophthalmol 251: 1541-1555, 2013.

15. Cheung CM, Awan MA and Sandramouli S: Prevalence and clinical findings of tamsulosin-associated intraoperative floppy-iris syndrome. J Cataract Refract Surg 32: 1336-1339, 2006.

16. Schwinn DA and Afshari NA: Alpha (1)-Adrenergic receptor antagonists and the iris: New mechanistic insights into floppy iris syndrome. Surv Ophthalmol 51: 501-512, 2006.

17. Wong AC and Mak ST: Finasteride-associated cataract and intraoperative floppy-iris syndrome. J Cataract Refract Surg 37: 1351-1354, 2011.

18. Chatziralli IP, Sergentanis TN, Papazisis L and Moschos MM: Risk factors for intraoperative floppy iris syndrome: A retrospective study. Acta Ophthalmol 90: e152-e153, 2012.

19. Pringle E and Packard R: Antipsychotic agent as an etiologic agent of IFIS. J Cataract Refract Surg 31: 2240-2241, 2015.

20. Unal M, Yücel I and Tenlik A: Intraoperative floppy-iris syndrome associated with chronic use of chloropromazine. Eye (Lond) 21: 1241-1242, 2007.

21. Altiaylik Ozer P, Altiparmak UE, Unlu N, Hazirolan DO, Kasim R and Duman S: Intraoperative floppy-iris syndrome: Comparison of tamsulosin and drugs other than alpha antagonists. Curr Eye Res 38: 480-486, 2013

22. Papadopoulos R and Bachariou A: Intraoperative floppy-iris syndrome associated with chronic intake of donepezil. J Cataract Refract Surg 33: 1997-1998, 2007.

23. Bilgin B, Ilhan D, Cetinkaya A and Ünal M: Intraoperative floppy iris syndrome associated with quetiapine. Eye (Lond) 27: 673, 2013.

24. Ford RL, Sallam A and Towler HM: Intraoperative floppy iris syndrome associated with risperidone intake. Eur J Ophthalmol 21: 210-211, 2011.

25. Gupta A and Srinivasan R: Floppy iris syndrome with oral imipramine: A case series. Indian J Ophthalmol 60: 136-138, 2012.

26. González-Martín-Moro J, González-López JJ,Zarallo-Gallardo J and Fernández-Miguel Y: Intraoperative floppy iris syndrome after treatment with duloxetine: Coincidence, association, or causality? Arch Soc Esp Oftalmol 90: 94-96, 2015 (In Spanish).

27. Asensio-Sánchez VM: Intraoperative floppy iris syndrome and warfarin: Coincidence or side effect? Arch Soc Esp Oftalmol 88: 160, 2013 (In English, Spanish).

28. Neff KD, Sandoval HP, Fernández de Castro LE, Nowacki AS, Vroman DT and Solomon KD: Factors associated with intraoperative floppy iris syndrome. Ophthalmology 116: 658-663, 2009.

29. Yu Y and Koss MC: Studies of alfa adrenoreceptor antagonists on simpathetic in rabbits. J Ocul Pharmacol Ther 19: 255-263, 2003.

30. Hovanesian JA and Chang DF: State of the art: Intraoperative floppy iris syndrome. Ocular Surg News U.S. Edition, March 10, 2008.

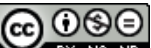

This work is licensed under a Creative Commons

Attribution-NonCommercial-NoDerivatives 4.0 International (CC BY-NC-ND 4.0) License. 